\title{
RS3PE ASSOCIATED WITH GIST TUMOR
}

Thais Miranda da Silva de Andrade ${ }^{1, \star}$, Marina Pereira Machadoํ․, Roberta Zawadzki Bueno ${ }^{1}$, Ana Cristina Boni Brandão Lenci Marques ${ }^{1}$, Thaíssa Antunes Mattar Valente ${ }^{1}$, Fabio Henrique Carneiro ${ }^{1}$, André Wilson de Lima Oliveira ${ }^{1}$, Maxwell Cássio de Albuquerque Pessoa ${ }^{1}$, Gabriel Caetano Pereira ${ }^{1}$, Eduardo Dos Santos Paiva ${ }^{1}$

1.Universidade Federal do Paraná, Curitiba (PR), Brazil.

${ }^{\star}$ Corresponding author: thais_.andrade@hotmail.com

\section{BACKGROUND}

Remitting seronegative symmetrical synovitis with pitting edema (RS3PE) is a rare inflammatory disease of unknown etiology, characterized by edema of the extremities and arthritis, which mainly affects patients over 50 years of age.

Rheumatoid factor and anti-CCP are usually negative. The syndrome can be isolated or related with other diseases. There are reports of paraneoplastic association with prostate, lung, lymphoproliferative and gastrointestinal tumors. This patient was diagnosed with gastrointestinal stromal tumor (GIST), for which there are no publications with a direct relation with RS3PE. Patients diagnosed with RS3PE usually present good response to corticosteroids, as was the case of our patient.

\section{CASE REPORT}

A 63-year-old man, previously hypertensive and heavy drinker was admitted for severe COVID-19 infection, needing intubation and hemodialysis. During hospitalization, he developed knee monoarthritis, and an arthrocentesis revealed cloudy synovial fluid, 7,429 leukocytes $/ \mu \mathrm{L}$, with negative gram stain and culture. He was treated as possible septic arthritis due to prolonged hospitalization, multiple infections and use of broad-spectrum antibiotic therapy, with improvement After medical discharge, the patient developed symmetrical polyarthritis of the hands, feet, ankles and knees, prolonged morning stiffness, pitting edema of hands and feet. An extensive investigation was carried out in the outpatient clinic and reactive arthritis, gout, and rheumatoid arthritis were excluded. Investigation of possible associated neoplasia was initiated, due to persistent iron deficiency anemia, weight loss, and melena. Diagnosis of GIST was made by computed tomography of the abdomen and upper digestive endoscopy, and it was surgically resected. The patient was treated with prednisone $10 \mathrm{mg} /$ day and showed improvement in the joint condition.

\section{CONCLUSION}

The association of RS3PE with GIST has never been described in the literature before. This rare association demonstrates the importance of screening for cancer in elderly patients who are suspected of having this syndrome.

\section{KEYWORDS}

RS3PE, GIST, Pitting edema, Seronegative symmetrical synovitis. 